\title{
Principally supplemented module and lifting property
}

\author{
Majid Mohammed* and Abd Ghafur \\ Faculty of Sciences and Technology Universiti Kebangsaan, School of Mathematical Science, Malaysia.
}

Accepted 12 April, 2013

\begin{abstract}
The purpose of this paper is to further study the concept of a very important module theory namely principally supplemented modules. Therefore, our work presents some properties of the relation between lifting property and principally supplemented module. Also we will focus on quasi-discrete module to study principally supplemented in general way. We prove that if $R$ be principal ideal ring and if $M$ be indecomposable $R$-module such that it has no maximal submodules $(\operatorname{Rad}(M)=M)$ then $M$ is principally supplemented module. Also we prove that if $M$ is indecomposable and local module then it is principally supplemented. Every projective module is self-projective therefore we prove that if $M$ projective and supplemented module then $M$ is principally supplemented.
\end{abstract}

Key words: Principally supplemented, $P$-hollow module, $f$-lifting module, quasi-discrete module.

\section{INTRODUCTION}

Throughout this paper all rings have the identity and modules are considered to be right modules. A principally supplemented modules generalizes principally lifting modules. A submodule $N$ of $M$ is called small in $M(N \ll$ $M)$ if for every submodule of $M, N+L=M$ then $L=M$. A submodule $N$ of $M$ is called a supplement of $K$ in $M$ if $N+$ $K=M$ and $N$ is minimal with respect to this property. A module $M$ is called supplemented if any submodule $N$ of $M$ has a supplement in $M$. A module $M$ is called a lifting (or satisfies $\left(D_{1}\right)$ ) if for any submodule $N$ of $M$, there exists a direct summand $K$ of $M$ such that $K \leq N$ and $(M /$ $M) \ll(M / M)$ equivalently, for every submodule $N$ of $M$ there exists submodules $K_{1}$ and $K_{2}$ of $M$ such that $M=K_{1}$ $\oplus K_{2}, K_{1} \leq N$ and $\left(N^{\cap} K_{2}\right) \ll K_{2}$, and the module $M$ has $\left(D_{2}\right)$ property if $N \leq M$ such that $(M / M)$ is isomorphic to a direct summand of $M$, then $N$ is a direct summand of $M$. A module $M$ is called $\left(D_{3}\right)$ if for every direct summands $K$ and $L$ of $M$ with $M=K+L, K^{n} L$ is a direct summand of $M$. A module $M$ is called amply supplemented if for any two submodules $A$ and $B$ of $M$ with $M=A+B$, there exists a supplement $P$ of $A$ in $M$ which is contained in $B$. Clearly, every amply supplemented module is supplemented. The concepts of hollow and hollow-lifting are very important to study a supplemented module. A module $M$ is called hollow if every proper submodule $N$ of $M$ is small in $M$ and a module $M$ is called hollow-lifting if every submodule $N$ of $M$ such that $(M / N)$ is hollow has a coessential submodule that is direct summand of $M$. According to Inankil et al. (2011) to conclude new concept is called semi-hollow ( $P$-hollow) which means any proper finitely generated submodule is small in $M$. A module $M$ is called $f$-lifting if and only if $M$ is principally lifting (or $P D_{1}$ ). Talebi (2007) indicated that an $R$-module $M$ is called $c$-f-lifting if every submodule of $M$ which contained coessentially in a finitely generated submodule lies above a direct summand and every lifting module is $f$ lifting. A module $M$ is called local if $M$ has largest submodule (that is, a proper submodule which contains all other proper submodules). Let $M$ be an $R$-module then $M$ is called a projective cover of $N$ if $M$ is a projective and there exists an epimorphism $f$ from $M$ into $N$ such that kernel $(f) \ll M$. A module $M$ is said to be quasi- discrete if $M$ satisfies the following properties $\left(D_{1}\right)$ and $\left(D_{3}\right)$. By Lomp (2000) we know $\left(D_{2}\right) \rightarrow\left(D_{3}\right)$. Therefore, 
every discrete module is quasi-discrete. A module $M$ is called coatomic, if every proper submodule of $M$ is contained in a maximal submodule of $M$; or equivalently, for a submodule $N$ of $M$, whenever $\operatorname{Rad}\left(\frac{M}{N}\right)=(M / N)$ then $M=N$. ÄOzcan and Pinar (2008) shows that every finitely generated and semisimple modules are coatomic. In this paper we study some properties of principally supplemented module by two concepts, which are namely lifting property and principally hollow module. Also we can study this matter by other module is called quasi-discrete to obtain a new results about principally supplemented.

\section{LIFTING AND P-SUPPLEMENTED MODULE}

A module $M$ is called indecomposable if $M \neq 0$, and it is not a direct sum of two nonzero submodules therefore if $R$ be principal ideal ring and $M$ be indecomposable $R$ module with $M=\operatorname{Rad}(M)$ then $M$ is principally supplemented module. Also since every indecomposable module is local then if $R$ is principal ideal ring and $M$ local module, then it is principally supplemented. A module $M$ is called hollow if every proper submodule $N$ of $M$ is small in $M$ and a module $M$ is called hollow-lifting if every submodule $N$ of $M$ such that $(M / N)$ is hollow has a coessential submodule that is direct summand of $M$. We know that every hollow module is semi-hollow, and hollow module is principally hollow, therefore every local module is principally supplemented. Now if $R$ principal ideal ring and $M$ be $R$-module such that $N$ submodule of $M$ and lies above a direct summand of $M$, then $M$ is $\left(D_{1}\right)$ module and this implies $M$ is principally supplemented. Also we can say:

(1) $P$-hollow modules need not be hollows. For example; considering the set $Q$ of all rational numbers as a $Z$ module $(Q=Z$ is not hollow, while $Q=N$ is not cyclic for all proper submodules $N$ of $Q$ ),

(2) Hollow modules are indecomposable modules, so direct sums of hollow modules are not hollows, according to Orhan et al. (2007), if $M=\oplus P_{i}, i \in l$, where the $P_{i}$ are non cyclic $P$-hollows for all $i \in I$, then $M$ is $P$-hollow.

\section{Definition 1}

A module $M$ is called lifting if for all $N$ submodule of $M$ there exists a direct decomposition $M=H^{\oplus}$ G such that $H$ submodule of $N$ and $(N \cap H)$ is small in $M$.

\section{Definition 2}

A module $M$ is principally lifting (or has $\left(P D_{1}\right)$ for short) if for all $m \in M, M$ has a decomposition $M=N \oplus S$ with $N$ submodule of $m R$ and $(m R \cap S) \ll M$. Therefore, $M$ is called principally supplemented module if it is $\left(P D_{1}\right)$.

\section{Definition 3}

An $R$-module $M$ is called principally supplemented (or ( $P$ supplemented) for short) in case of, for each $m \in M$, if $M$ $=m R+N$ then $m R$ contains a supplement of $N$ in $M$. For a submodule $B$ of $M, M$ is $B$-supplemented if $A$ contains a supplement of $B$ in $M$ whenever $M=B+A$. Equivalently of (Definition 3) a module $M$ is said to be principally supplemented if for any cyclic submodule has a supplement in $M$. If $M$ has a largest proper submodule, that is, a proper submodule which contains all other proper submodules, then $M$ is called a local module. Therefore a local module is always indecomposable. Let $M$ be an $R$-module if $M=\operatorname{Rad}(M)$ then $M$ is semi-hollow module. Let $M$ be a module and it has $\left(P D_{1}\right)$ over a principal ideal ring $R$. If $M=X+m R$, then $m R$ contains a supplement of $X$ in $M$.

\section{Definition 4}

A non zero module $M$ is called principally hollow ( $P$ hollow) or semi-hollow if every proper cyclic submodule is small in $M$.

\section{Lemma 1}

Let $M$ be an $R$-module. If $M$ be indecomposable then the following are equivalent:

(1) $M$ has $\left(P D_{1}\right)$,

(2) $M$ is a $P$-hollow module.

\section{Proof}

By the defining condition of $\left(P D_{1}\right)$.

\section{Theorem 1}

Let $M$ an $R$-module such that $M$ is finite uniform dimensional and $\left(P D_{1}\right)$ then $M$ is a direct sum of $P$-hollow submodules.

\section{Proof}

The proof will be by induction on the uniform dimension of $M$. If $N \cdot \operatorname{dim}(M)=1$, then $M$ is an indecomposable module; and hence every $M$ has $\left(P D_{1}\right)$ then $M$ is a $P$ hollow module, by Orhan et al. (2007) Lemma 1. Now let $2 \leq(N \cdot \operatorname{dim}(M))=n<\infty$. Since $M$ is not a $P$-hollow 
module, then $\exists m \in M \ni m R$ is not small and proper submodule of $M$. Since we have $\left(P D_{1}\right)$, then $M=L_{1} \oplus L_{2}$ , $\ni L_{1} \leq m R$ and $L_{2} \cap m R \ll M$. Therefore $L_{1}$ and $L_{2}$ have uniform dimensions less than $n$, and hence by induction, $\forall L_{1}$ and $L_{2}$ is a direct sum of $P$-hollow submodules.

\section{Theorem 2}

Let $M=A^{\oplus} B$ is an $R$-module, where $A$ is simple and $B$ has a composition series $0 \leq K \leq B$, then $M$ has $\left(P D_{1}\right)$.

\section{Proof}

Let $N$ be a nonzero proper cyclic submodule of $M$. We assume that $N=(r+s) R$, where $0 \neq r^{\in} R_{1}$ and $0^{\neq} s^{\in}$ $S_{1}$. Then $N+R_{1}=R_{1} \oplus s R$, and that $s R$ is either $K$ or $S_{1}$. Since $R_{1}$ is simple, then either $R_{1} \leq N$ or $R_{1} \cap N=0$. If $R_{1}$ $\leq N$, then $s R=K$ and $N=R_{1} \oplus K$ (due to $R_{1}$ a proper submodule of $M$ ), $\ni R_{1} \leq M$ and $K{ }^{\ll} M$. Also, if $R_{1} \cap N=0$ then $N \oplus R_{1}=R_{1} \oplus s R$. Let $s R=S_{1}$, then $N \leq M$, and if $s R=K$, then $N \cong K$ is a simple module. But $M=N+S_{1}$ with $N \leqslant S_{1}$; and hence $N^{n} S_{1}=0$, and this implies $N \leq$ $M$. Therefore $M$ has $\left(P D_{1}\right)$.

\section{Remark 1}

A principally supplemented modules generalizes principally lifting modules and here we show that modules which satisfy the condition $\left(P D_{1}\right)$ need not be even principally supplemented ( $P$-supplemented), but over principal ideal rings they are $P$-supplemented.

\section{Theorem 3}

Let $R$ be principal ideal ring and let $M$ be indecomposable $R$-module. If $M$ has no maximal submodule then $M$ is principally supplemented module.

\section{Proof}

Since $M$ has no maximal submodule then $M$ is injective module and this means $(\operatorname{Rad}(M)=M)$ therefore $M$ is semi-hollow and this imply every proper finitely generated submodule is small in $M$. By this fact we can say that every proper cyclic submodule is small in $M$, then $M$ is principally hollow module and then by Lemma 1 we get $M$ is $\left(P D_{1}\right)$ and since $R$ is principal ideal ring therefore $M$ is principally supplemented.

\section{Theorem 4}

Let $R$ be principal ideal ring and let $M$ has indecomposable property. If $M$ is local, then it is principally supplemented.

\section{Proof}

Let $M$ be local module, then $M$ has largest submodule or a proper submodule which contains all other submodules. Now we can say that $M$ is hollow module, therefore $M$ is semi-hollow then every proper finitely generated submodule is small in $M$ and this mean $M$ is principally hollow and with indecomposable property imply $M$ is $\left(P D_{1}\right)$ and $R$ is principal ideal ring and hence $M$ is principally supplemented module.

\section{Proposition 1}

Let $M$ be a module over principal ideal ring $R$. Then the following statements are equivalent:

1. $M$ is injective module,

2. $\operatorname{Rad}(M)=M$,

3. $M$ is semi-hollow module,

4. $M$ is principally hollow module ( $P$-hollow),

5. $M$ has principally lifting,

6. $M$ is principally supplemented.

A module $M$ is called f-lifting if and only if $M$ is principally lifting (or $P D_{1}$ ) and $M$ is called c-f-lifting if every submodule of $M$ which contained coessentialy in a finitely generated submodule lies above a direct summand, therefore every lifting module is f-lifting. A ring $R$ is called $V$-ring if every simple R-module is injective. Then every Noetherian and semisimple $R$-module $M$ over $V$-ring is $c$ $f$-lifting and since lifting module is hollow-lifting and so is $c$-f-lifting therefore if $R$ be a principal ideal ring and $M$ is a lifting module, then $M$ is principally supplemented. Moreover, if $M$ is c-f-lifting, this implies $M$ is finitely lifting if and only if $M$ is lifting and so if $M$ is f-lifting, then it is $\left(P D_{1}\right)$. For any principal ideal ring $R$ if a module $M$ is hollow-lifting, then $M$ is principally supplemented.

\section{Corollary 1}

Let $R$ be principal ideal ring. If $M$ is $c$-f-lifting module, then $M$ is principally supplemented.

\section{Corollary 2}

For any principal ideal ring $R$ if a module $M$ is hollowlifting, then $M$ is principally supplemented. 


\section{Proof}

Since $M$ is hollow-lifting, then $M$ is $c$-f-lifting and so lifting and then $M$ is $f$-lifting and finally $M$ is $\left(P D_{1}\right)$, then $M$ principally supplemented.

\section{Corollary 3}

Let $R$ be a simple ring and $M$ is $\oplus$-supplemented, then $M$ $=\operatorname{Rad}(M)$ and so $M$ is principally supplemented.

\section{Examples 1}

1. $Z_{4}$ as $Z$-module is $f$-hollow-lifting module,

2. $Z$ as $Z$-module is not $f$-hollow-lifting module,

3. Every hollow-lifting module is f-hollow-lifting. But the converse is not true in general, for example, consider $Q$ as Z- module is f-hollow-lifting but not hollow-lifting.

Let $M, N$ and $P$ be $R$-modules then $P$ is projective if for every epimorphism $f: M \rightarrow N \rightarrow 0$ and homomorphism $g: P \rightarrow N$ there is a homomorphism $h: P \rightarrow M$ such that $f_{0} h$ $=g$. Also $M$ is called a projective cover of $N$ if $M$ is a projective and there exists an epimorphism $f$ from $M$ into $N$ such that kernel of $f$ is small in $M$. A module $M$ is called $\pi$-projective if there exists $\beta: M \rightarrow M$ is endomorphism of $M$ such that image of $\beta$ is submodule of $N$ and image of (1$\beta$ ) is submodule of $L$ such that $N$ and $L$ submodules of $M$ and $M=L+N$ or $M$ is $\pi$-projective $R$-module if and only if the epimorphism $\beta$ from ( $U$ into $M$ defined by $\beta((u, v)$ ) $=u$ $+v$ splits. Recall that a module $M$ is called a lifting module (or satisfies $\left(D_{1}\right)$ ) if for any submodule $N$ of $\mathrm{M}$, there exists a direct summand $\mathrm{K}$ of $M$ such that $K \leq N$ and $(N / K) \ll(M / K)$. Now we can say if $R$ principal ideal ring and $M$ be $R$-module such that submodule of $M$ and lies above a direct summand of $M$, then $M$ is $\left(D_{1}\right)$-module and this implies $M$ is principally supplemented. Also if $M$ $=A \oplus B$ is an $R$-module, where $A$ is simple and $\mathrm{B}$ has a composition series $0 \leq K \leq B$, then $M$ has $\left(P D_{1}\right)$.

\section{Proposition 2}

Let $M$ be a projective module over principal ideal ring $R$. If $N$ finitely generated submodule of $M$ such that is hollow and has a projective cover, then $M$ is principally supplemented.

\section{Proof}

Let $N$ be finitely generated submodule of $M$ and $(M / N)$ is hollow. Let is natural a mapping from $M$ into $(M / N)$ Since $(M / N)$ has a projective cover this mean there exists a decomposition $M=M_{1} \oplus \mathrm{M} 2$ such that $\beta \mid M_{2}: M_{2} \rightarrow(M)$
$N$ is a projective cover and $M_{1} \subseteq \operatorname{ker}(\beta)$. Therefore $M_{1}$ subset of $N$, also $\operatorname{ker}\left(\beta / M_{2}\right)=N \cap M_{2}$ is small in $M$. Thus $M$ is f-hollow-lifting and this implies $M$ is f-lifting and so $M$ is $\left(P D_{1}\right)$ and hence principally supplemented module.

\section{Proposition 3}

Let $M$ be an module over principal ideal ring $R$. If $M$ satisfy the following:

1. $M$ is hereditary module,

2. $\operatorname{Rad}(M) \ll M$,

3. $M$ is Rad-supplemented.

Then $M$ is lifting module and so is principally supplemented.

\section{Corollary 4}

Let $R$ be a simple ring and $M$ is $\oplus$-supplemented, then $M$ $=\operatorname{Rad}(M)$ and so $M$ is principally supplemented.

\section{QUASI-DISCRETE AND P-SUPPLEMENTED MODULE}

There are many properties which related to quasidiscrete module and so implies principally supplemented module as:

$\left(D_{2}\right)$ : A module $M$ is called $\left(D_{2}\right)$ - module if $N$ submodule of $M$ such that $(M / M)$ is isomorphic to a direct summand of $M$, then $N$ is a direct summand of $M$.

$\left(D_{3}\right)$ : A module $M$ is called $\left(D_{3}\right)$ if for every direct summands $K$ and $L$ of $M$ with $M=K+L, K \cap L$ is a direct summand of $M$.

$\left(D_{12}\right)$ : A module $M$ is called $\left(D_{12}\right)$ if for every submodule $K$ of $M$ there exist a direct summand $N$ of $M$ and an epimorphism $\delta: M=N \rightarrow(M / K)$ with $\operatorname{Ker}(\delta) \ll(M / N)$.

$\left(T_{1}\right)$ if for every submodule $K$ of $M$, where $(M / K)$ is isomorphic to a coclosed submodule of $M$, every homomorphism $\quad \mu: M \rightarrow(M / K)$ lifts to a homomorphism $\beta: M \rightarrow M$.

Now from above properties we can say a module $M$ is said to be quasi-discrete if $M$ satisfies the following properties $\left(D_{1}\right)$ and $\left(D_{3}\right)$ and since $\left(D_{2}\right) \rightarrow\left(D_{3}\right)$. Therefore, $\left(D_{1}\right)$-property with $\left(D_{2}\right)$-property implies quasi-discrete module. Also, $M$ is called hollow if it is an indecomposable lifting module. Now $M$ is called amply supplemented if for any two submodules $L$ and $K$ of $M$ such that $L+K=M$ and $K$ contains a supplement of $L$ in $M$.

\section{Theorem 5}

Let $R$ be principal ideal ring and let $M$ be quasi-discrete 
module over $R$, then $M$ is principally supplemented module.

\section{Corollary 5}

Let $R$ be principal ideal ring and let $M$ be an $R$-module. If $M$ is lifting and has $\left(D_{3}\right)$ property, then $M$ is principally supplemented module.

\section{Proof}

Since $M$ is lifting and has $\left(D_{3}\right)$ property then $M$ is supplemented and $\pi$-projective then $M$ is quasi-discrete and so $M$ is $\left(P D_{1}\right)$ module with $R$ principal ideal ring implies $M$ is principally supplemented module.

\section{Proposition 4}

Let $R$ be principal ideal ring and let $\mathrm{M}$ be an $R$-module. If $M=P_{1} \oplus P_{2}$ such that $M$ is lifting and $P_{1}$ and $P_{2}$ are relatively projective then $M$ is principally supplemented.

\section{Proof}

Since $M$ is lifting module then if $L+K=M$ such that $L, K$ submodules of $M$, then $L$ lies above a direct summand of $M_{1}, M=M_{1} \oplus M_{2}, M_{1}+K=M$ and since $M_{2}$ is relatively projective then there is a mappings $\pi: M \rightarrow M_{1}$ and $\mu: M_{1}$ $\rightarrow M$ such that $g=\pi \mu$ is an endomorphism of $M$ then $M$ is $\pi$-projective. Also since $M$ is lifting then $M$ is supplemented with $\pi$-projective implies $M$ is quasidiscrete then $M$ is $\left(P D_{1}\right)$ module but $R$ is principal ideal ring then $M$ is principally supplemented.

\section{Corollary 6}

Let $R$ be principal ideal ring and let $M$ be an $R$-module. If $M$ is projective then $M$ is principally supplemented.

\section{Proof}

Since $M$ projective module then $M$ is self projective and so $M$ is discrete module and every discrete module is quasi-discrete then $M$ is $\left(P D_{1}\right)$, but $\mathrm{R}$ principal ideal ring implies that $M$ is principally supplemented.

\section{Corollary 7}

If $M$ is projective and supplemented module over principal ideal ring then $M$ is principally supplemented module. Recall that an $R$-module $M$ is quasi-discrete if it is hollowlifting and if every module having no hollow factor modules is a hollow-lifting then $M$ is principally supplemented. If $M$ is indecomposable and hollow module then $M$ is hollow-lifting.

\section{Lemma 2}

Let $M=\oplus N_{\mathrm{i}}, \mathrm{i}=1, \ldots, n$ with all Ni hollow. If $M$ has $\left(D_{3}\right)$, then the following statements are equivalent:

(1) $M$ is lifting;

(2) $M$ is quasi-discrete;

(3) $M$ is hollow-lifting; $M$ is lifting;

(4) $\mathrm{Ni}$ is $N_{\mathrm{j}}$-projective for all $\mathrm{i} \neq \mathrm{j}$.

\section{Corollary 8}

For any module $M$ over principal ideal ring. If $M$ is local module with indecomposable property then $M$ is principally supplemented.

\section{Proof}

Since $M$ is local module then $M$ is hollow and with indecomposable property implies $M$ is hollow-lifting and then $M$ is lifting and hence $M$ is Quasi-discrete and this implies $M$ is $\left(P D_{1}\right)$ with $R$ principal ideal ring we obtain $M$ is principally supplemented.

\section{Theorem 6}

Let $M$ be an $R$-module such that $M$ satisfy the following conditions:

1- $M$ is coatomic module.

2- $M$ is semisimple module.

3- $\operatorname{Rad}(M)=0$.

Then $M$ is principally supplemented.

\section{Theorem 7}

Let $M$ be a module with $\left(P D_{1}\right)$. Then every indecomposable cyclic submodule $N$ of $M$ is either small in $M$ or a summand of $M$.

\section{Proof}

We know that $N=N \oplus S$ with $N \leq M$ and $S \leq M$. Since $N$ is indecomposable, we have either $N=N$ or $N=S$. Let $M$ be a module over principal ideal ring $R$ and if we have the following facts:

1. $D_{1}+D_{2} \Rightarrow M$ Discrete module, 
2. $D_{1}+D_{2} \Rightarrow D_{1}+T_{1}$,

3. $D_{1}+D_{3} \Rightarrow M$ Quasi-Discrete module

4. $D_{2} \Rightarrow D_{3}$ module.

5. $\mathrm{T} 1 \Rightarrow D_{2}$ module.

6. $D_{1} \Rightarrow$ amply supplemented and $D_{12}$ module.

Therefore we can conclude the following new implications:

$D_{1}+D_{2} \Rightarrow M$ Quasi-Discrete $\Rightarrow M$ P-supplemented.

$D_{1}+T_{1} \Rightarrow$ M Quasi-Discrete $\Rightarrow$ M P-supplemented.

$M$ Amply supplemented $+D_{12}+T_{1} \Rightarrow M$ Discrete module $\Rightarrow M$ Quasi-Discrete module $\Rightarrow M$ P-supplemented.

\section{REFERENCES}

ÄOzcan AC, Pinar A (2008). "Generalization of Semiregular and Semiperfect Modules”. Algebra Colloq. 15(4):667-680.
Inankil H, Halıcıoglu S, Harmanci A (2011). "A generalization of supplemented modules". Algebra Discrete Math. 11(1):59-74.

Lomp C (2000). "On Dual Goldie Dimension". angefertigt am Mathematischen Institut, der Heinrich-Heine Universitat Dusseldorf.

Orhan N, Tutuncu DK, Tribak R (2007). "On Hollow-Lifting Modules". Taiwanese J. Math. 11(2):545-568.

Talebi Y (2007). "A Generalization of Lifting Modules". J. Contemp. Math. Sci. 2(22):1069-1075. 